\title{
MicroRNA-195 inhibits cell proliferation, migration and invasion by targeting defective in cullin neddylation 1 domain containing 1 in cervical cancer
}

\author{
JINYAN ZHONG, HUI YUAN, XIANGQIAN XU and SHOUFANG KONG
}

Department of Gynecology, Affiliated Qingdao Hiser Hospital of Qingdao University, Qingdao, Shandong 266000, P.R. China

Received May 30, 2017; Accepted April 26, 2018

DOI: $10.3892 /$ ijmm.2018.3660

\begin{abstract}
MicroRNAs (miRs), a class of small non-coding RNAs, have been demonstrated to perform promoting or suppressive roles in various types of human malignancy. Deregulation of miR-195 has been observed in numerous types of human cancer, including cervical cancer; however, the detailed molecular mechanism of miR-195 underlying the malignant progression of cervical cancer remains largely unclear. In the present study, miR-195 was significantly downregulated in cervical cancer tissue samples compared with adjacent non-tumor tissue samples, and the reduced expression level of miR-195 was associated with node metastasis and an advanced clinical stage in cervical cancer. Furthermore, the patients with low miR-195 expression levels demonstrated shorter survival times when compared with those with high miR-195 expression levels. In vitro experiments indicated that miR-195 exerted suppressive effects on the proliferation, migration and invasion of cervical cancer cells. Luciferase reporter gene assay identified defective in cullin neddylation 1 domain containing 1 (DCUN1D1) as a novel target gene of miR-195 and the expression level of DCUN1D1 was identified to be negatively regulated by miR-195 in cervical cancer cells. DCUN1D1 was significantly upregulated in cervical cancer, with a negative correlation to miR-195 expression. Furthermore, upregulation of DCUN1D1 was associated with the malignant progression and poor prognosis of cervical cancer. DCUN1D1 overexpression attenuated the suppressive effects of miR-195 on the malignant phenotypes of cervical cancer cells. Notably, the expression levels of miR-195 were significantly lower in HeLa [human papilloma virus (HPV) $18^{+}$] and $\mathrm{SiHa}\left(\mathrm{HPV} 16^{+}\right)$cells compared with those in $\mathrm{C} 33 \mathrm{~A}\left(\mathrm{HPV}^{-}\right)$cells, and knockdown of E6 using small interfering RNA significantly increased the miR-195 expression while the DCUN1D1 expression level was
\end{abstract}

Correspondence to: Dr Hui Yuan, Department of Gynecology, Affiliated Qingdao Hiser Hospital of Qingdao University, 4 Renmin Road, Qingdao, Shandong 266000, P.R. China

E-mail: doctoryuanhui@163.com

Key words: microRNA, cervical cancer, defective in cullin neddylation 1 domain containing 1 , metastasis reduced in HeLa and SiHa cells. Thus, these findings indicate that miR-195 exerts a suppressive role in cervical cancer by targeting DCUN1D1. Therefore, miR-195 may present as a potential therapeutic candidate for cervical cancer.

\section{Introduction}

Cervical cancer is the third most common type of cancer and the fourth most frequent cause of cancer-associated mortality in women worldwide (1). There are $~ 530,000$ novel cervical cancer cases every year (1). Furthermore, the majority of these cervical cancer cases occur in developing countries, including China $(1,2)$. Due to its recurrence and metastasis, the prognosis for cervical cancer patients is unsatisfactory, although great improvements have been made in surgery combined with radiotherapy and/or chemotherapy (2). Therefore, novel therapeutic targets for the treatment of cervical cancer are urgently required.

MicroRNAs (miRs), a class of small non-coding RNAs, regulate gene expression by directly binding to the 3'-untranslated region (UTR) of their target messeger (m)RNA, causing translation repression or messenger (m)RNA degradation (3-5). In the past decade, a large number of miRs have been identified to performed important roles in various cellular biological processes, such as cell proliferation, apoptosis, migration, invasion and tumorigenesis (6-8). During the development and malignant progression of cervical cancer, many miRs have been demonstrated to be significantly deregulated and act as oncogenes or tumor suppressors, such as miR-27 (9), miR-124 (10), miR-140 (11) and miR-200 (12). Recently, miR-195 was identified to exert a tumor suppressive role in various common types of human cancer $(13,14)$. For example, miR-195 was observed to inhibit glucose uptake and proliferation of human bladder cancer cells by targeting solute carrier family 2 member 3 (13). In addition, miR-195 was found to inhibit the proliferation, migration and invasion of cervical cancer cells by targeting SMAD family member 3 (Smad3), cyclin D2 (CCND2), MYB proto-oncogene, transcription factor (MYB) and cyclin D1 (15-17). Thus, miR-195 may be key in the development and progression of cervical cancer; however, whether other target genes of miR-195 also contribute to cervical cancer remains largely unclear.

In the current study, the clinical significance of miR-195 expression levels in cervical cancer, as well as the regulatory mechanism of miR-195 underlying cervical cancer progression 
were investigated. The data indicate that miR-195 performs a suppressive role in cervical cancer by targeting DCUN1D1.

\section{Materials and methods}

Clinical tissue samples. The present study was approved by the Ethics Committee of Affiliated Qingdao Hiser Hospital of Qingdao University (Qingdao, China). A total of 72 cervical cancer tissue samples and matched adjacent normal tissue samples were obtained from the Department of Gynecology at the Qingdao Hiser Hospital between April 2010 and March 2012. Written informed consent was obtained from each patient involved in the study. All patients had not received radiation therapy or chemotherapy prior to surgery. The survival time was counted from the date of surgery to the follow-up at 5 years, or the date of death. The tissue samples were stored immediately in liquid nitrogen following surgical resection and stored at $-80^{\circ} \mathrm{C}$ prior to use.

Cell culture and transfection. Certain common human cervical cancer cell lines [HeLa (ATCC ${ }^{\circledR}$ CRM-CCL-2 ${ }^{\mathrm{TM}}$ ); $\mathrm{SiHa}$ (ATCC ${ }^{\circledR} \mathrm{HTB}-35^{\mathrm{TM}}$ ); C33A (ATCC ${ }^{\circledR} \mathrm{HTB}-31^{\mathrm{TM}}$ )] were purchased from the American Type Culture Collection (Manassas, VA, USA). Cells were cultured in Dulbecco's modified Eagle's medium (DMEM) supplemented with $10 \%$ fetal bovine serum (FBS) (both from Thermo Fisher Scientific, Inc., Waltham, MA, USA) and maintained at $37^{\circ} \mathrm{C}$ in a humidified atmosphere containing $5 \% \mathrm{CO}_{2}$. Cell transfection was performed using Lipofectamine 2000 (Thermo Fisher Scientific, Inc.) according to the manufacturer's instructions. Following transfection for $48 \mathrm{~h}$, the expression level of miR-195 or DCUN1D1 was evaluated.

RNA extraction and reverse transcription-quantitative polymerase chain reaction $(R T-q P C R)$. Total RNA was extracted from the tissue samples and cells using TRIzol Reagent (Thermo Fisher Scientific, Inc.) according to the manufacturer's instructions. DNase (Takara Biotechnology Co., Ltd., Dalian, China) treatment was used to remove genomic DNA, according to the manufacturer's instructions. For miR expression detection, a SYBR-Green PrimeScript miRNA RT-PCR kit (cat. no. RR716; Takara Biotechnology Co., Ltd.) was used to perform the RT-qPCR, according to the manufacturer's instruction. For mRNA expression detection, a SYBR-Green PrimeScript miRNA RT-PCR kit (cat. no. RR716; Takara Biotechnology Co., Ltd.) was used to perform the RT-qPCR, according to the manufacturer's instructions. U6 or GAPDH served as the internal reference for miR or mRNA expression, respectively. The primer sequences were as follows: Forward, 5'-AGGATCATTGGACAGGAAGAAGT-3' and reverse, 5'-TGCCAGGTCATCACAGAACTG-3' for DCUN1D1; and forward, 5'-GGAGCGAGATCCCTCCAAAAT-3' and reverse, 5'-GGCTGTTGTCATACTTCTCATGG-3' for GAPDH. The primers for miR-195 (HmiRQP0283) and U6 (HmiRQP9001) were purchased from Guangzhou FulenGen, Co., Ltd. (Guangzhou, China). The reaction conditions were as follows: $95^{\circ} \mathrm{C}$ for $10 \mathrm{~min}$, and 45 cycles of $95^{\circ} \mathrm{C}$ for $15 \mathrm{sec}$ and $60^{\circ} \mathrm{C}$ for $15 \mathrm{sec}$. The relative expression was analyzed according to the $2^{-\Delta \Delta \mathrm{Cq}}$ method (18). This experiment was repeated three times.

MTT assay. The HeLa and SiHa cell suspension $\left(5 \times 10^{4}\right.$ cells/well) was plated in a 96-well plate and cultured at $37^{\circ} \mathrm{C}$ for $0,24,48$ or $72 \mathrm{~h}$, respectively. Subsequently, MTT $(10 \mu \mathrm{l} ; 5 \mathrm{mg} / \mathrm{ml})$ was added to each well and the cells were incubated at $37^{\circ} \mathrm{C}$ for $4 \mathrm{~h}$. The supernatant was then removed and $100 \mu \mathrm{l}$ dimethyl sulfoxide was added to each well. The absorbance at a wavelength of $570 \mathrm{~nm}$ was determined using a Model 680 Microplate Reader (Bio-Rad Laboratories, Inc., Hercules, CA, USA). The experiment was repeated three times.

Western blot analysis. Tissue samples and cells were lysed in cold radioimmunoprecipitation assay buffer (Thermo Fisher Scientific, Inc.). A BCA Protein Assay kit (cat. no. 23225; Pierce; Thermo Fisher Scientific, Inc.) was used to determine the concentration of protein according to the manufacturer's instructions. Protein $(50 \mu \mathrm{g})$ was then separated using $10 \%$ SDS-PAGE (50 V for $1 \mathrm{~h}$ followed by $400 \mathrm{~mA}$ for $4 \mathrm{~h}$ ) and transferred to a polyvinylidene fluoride (PVDF) membrane (Thermo Fisher Scientific, Inc.). After blocking in 5\% non-fat dried milk in Dulbecco's phosphate-buffered saline (DPBS; Thermo Fisher Scientific, Inc.) at room temperature for $3 \mathrm{~h}$, the PVDF membrane was incubated with rabbit anti-human DCUN1D1 monoclonal antibody (1:50; cat. no. ab181233), or rabbit anti-human GAPDH monoclonal antibody (1:50; cat. no. ab181602) (both from Abcam, Cambridge, MA, USA) for $3 \mathrm{~h}$ at room temperature. After washing with DPBS three times, the membrane was incubated with goat anti-rabbit IgG (1:5,000; cat. no. ab6721; Abcam) for $1 \mathrm{~h}$ at room temperature. After washing with DPBS three times, an Enhanced Chemiluminescence (ECL) Western Blotting kit (cat. no. 32109; Pierce; Thermo Fisher Scientific, Inc.) was used to detect the immune complex on the PVDF membrane according to the manufacturer's instructions. Image-Pro Plus software 6.0 (Media Cybernetics, Inc., Rockville, MD, USA) was used to analyze the protein expression. GAPDH served as an internal control and the experiment was repeated three times.

Wound healing assay. HeLa and SiHa cells ( $10^{5}$ cells) were seeded in a 6-well plate and cultured to confluence. The cell monolayer was scraped using a $200 \mu 1$ pipette tip to generate a wound, which was then washed with DMEM two times. Cells were cultured in DMEM with $3 \%$ FBS at $37^{\circ} \mathrm{C}$ for $48 \mathrm{~h}$. Cells were subsequently photographed under a light microscope (Olympus Corporation, Tokyo, Japan) at 0 and $48 \mathrm{~h}$ after wounding. The experiment was repeated three times.

Transwell assay. HeLa and SiHa cells were plated into the top side of a Transwell chamber pre-coated with Matrigel (BD Biosciences, San Jose, CA, USA). The lower chamber contained DMEM with $10 \%$ FBS as an attractant. Following incubation at $37^{\circ} \mathrm{C}$ for $24 \mathrm{~h}$, the upper surface of the Transwell chamber was scraped using cotton swabs. The invaded cells on the lower surface of the Transwell chamber were fixed with ethanol, stained for $10 \mathrm{~min}$ at room temperature using $0.05 \%$ Crystal Violet (Sigma-Aldrich; Merck KGaA, Darmstadt, Germany) and counted under a light microscope. The experiment was repeated three times.

Bioinformatics prediction. TargetScan (www.targetscan.org), miRDB (www.mirdb.org) and DIANAmT (www.microrna.gr) were applied to predict the putative target genes of miR-195, according to the manufacturer's instruction. 
Table I. Association between miR-195 expression level and clinicopathological characteristics of patients with cervical cancer.

\begin{tabular}{|c|c|c|c|c|}
\hline Variable & Patients $(n=72)$ & Low miR-195 $(n=46)$ & High miR-195 $(n=26)$ & P-value \\
\hline Age (years) & & & & 1.000 \\
\hline$<55$ & 27 & 19 & 8 & \\
\hline$\geq 55$ & 45 & 37 & 18 & \\
\hline Tumor size $(\mathrm{cm})$ & & & & 0.623 \\
\hline$\leq 4$ & 44 & 27 & 17 & \\
\hline$>4$ & 28 & 19 & 9 & \\
\hline Differentiation & & & & 0.165 \\
\hline Well to moderate & 53 & 31 & 22 & \\
\hline Poor & 19 & 15 & 4 & \\
\hline Clinical stage & & & & 0.043 \\
\hline I-II & 47 & 26 & 21 & \\
\hline III-IV & 25 & 20 & 5 & \\
\hline Lymph node metastasis & & & & 0.008 \\
\hline No & 49 & 27 & 22 & \\
\hline Yes & 23 & 20 & 3 & \\
\hline Distant metastasis & & & & 0.083 \\
\hline No & 62 & 37 & 25 & \\
\hline Yes & 10 & & 1 & \\
\hline
\end{tabular}

Luciferase activity assay. The wild-type (WT) DCUN1D1 3'-UTR containing the binding sites of miR-195 was amplified and subcloned into the pmirGLO luciferase reporter vector (Promega Corporation, Madison, WI, USA). The mutant-type (MuT) DCUN1D1 3-UTR lacking complementarity with miR-195 binding sites was generated using the QuickChange Site-Directed Mutagenesis kit (cat. no. 200518 Stratagene; Agilent Technologies, Inc., Santa Clara, CA, USA) according to the manufacturer's instructions, which was also subcloned into the pmirGLO luciferase reporter vector. HeLa and SiHa cells $\left(5 \times 10^{4}\right)$ were seeded in 24-well plates and co-transfected with WT or MuT 3'-UTR vectors and miR-195 mimics or miR-NC using Lipofectamine 2000. After $48 \mathrm{~h}$, the cells were assayed for luciferase activity using the Dual-Luciferase Reporter Assay System (Promega Corporation), according to the manufacturer's instructions. The firefly luciferase activities were normalized to Renilla luciferase activity. This experiment was repeated three times.

Statistical analysis. Data were presented as means \pm standard deviation. SPSS 17.0 statistical software (SPSS, Inc., Chicago, IL, USA) was used to conduct the statistical analysis. An independent, two-tailed Student's t test and one-way ANOVA were performed to compare the difference. The association between the expression level of miR-195 or DCUN1D1 and the clinicopathological characteristics of cervical cancer patients were examined using the $\chi^{2}$ test or Fisher's exact test. Survival analysis was evaluated using the Kaplan-Meier method. The correlation between the miR-195 and DCUN1D1 expression in cervical cancer was assessed using Pearson's correlation analysis. $\mathrm{P}<0.05$ was considered to indicate a statistically significant difference.

\section{Results}

Downregulation of miR-195 is associated with aggressive progression in cervical cancer. In the present study, the expression levels of miR-195 were examined using RT-qPCR in cervical cancer and adjacent non-tumor tissue samples. The data indicated that the expression levels of miR-195 were significantly decreased in cervical cancer tissue samples compared with adjacent non-tumor tissue samples (Fig. 1A). The clinical significance of miR-195 expression levels in cervical cancer was then evaluated. According to the mean value of miR-195 expression levels (1.52), these cervical cancer patients were divided into high and low miR-195 expression groups. As presented in Table I, the low expression level of miR-195 was significantly associated with node metastasis and an advanced TNM clinical stage in cervical cancer. In addition, as all of the cervical cancer patients involved in the present study were high-risk human papilloma virus (HPV)-positive, no correlation was observed between the miR-195 expression level and high-risk HPV infection (data not shown).

Downregulation of miR-195 predicts poor prognosis of patients with cervical cancer. The prognostic value of miR-195 expression levels in cervical cancer was then evaluated. The correlation between miR-195 expression and overall survival time of cervical cancer patients was examined using the Kaplan-Meier method. As presented in Fig. 1B, patients with low expression levels of miR-195 demonstrated shorter survival times compared with those with high expression levels of miR-195. Therefore, downregulation of miR-195 may predict poor prognosis of patients with cervical cancer. 
A

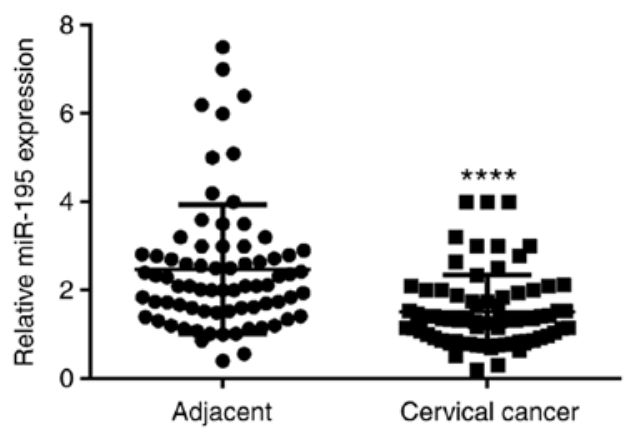

B $\quad-$ High miR-195 expression

- Low miR-195 expression

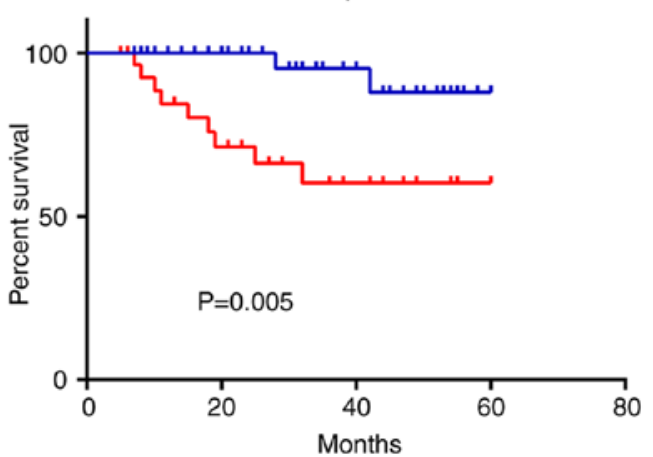

Figure 1. Downregulation of miR-195 in cervical cancer. (A) Reverse transcription-quantitative polymerase chain reaction was performed to evaluate the miR-195 expression levels in a total of 72 cervical cancer tissue samples and matched adjacent healthy tissue samples. ${ }^{* * * *} \mathrm{P}<0.0001$ vs. adjacent. (B) According to the mean value of miR-195 expression levels (1.52), the cervical cancer patients were divided into a high and a low miR-195 expression group. Low expression levels of miR-195 were associated with a shorter survival time of cervical cancer patients. The experiments were repeated three times. miR, microRNA.

miR-195 exerts suppressive effects on the proliferation, migration and invasion of cervical cancer cells. Subsequently, the effects of miR-195 on the malignant phenotypes of cervical cancer cells were investigated. An miR-195 mimic was used to transfect HeLa and SiHa cells. Following transfection, the miR-195 expression levels were significantly upregulated compared with the miR-negative control (NC) group (Fig. 2A). The MTT assay data further indicated that the miR-195-overexpressing cells demonstrated reduced proliferation when compared with those in the miR-NC group (Fig. 2B). These findings indicate that miR-195 may exert suppressive effects on cervical cancer growth. The role of miR-195 in the regulation of cervical cancer cell migration and invasion was then evaluated. Data from the wound healing and Transwell assays indicated that ectopic expression of miR-195 significantly reduced the migration and invasion of HeLa and SiHa cells (Fig. 3). These findings indicate that miR-195 may have a suppressive role in cervical cancer metastasis.

DCUN1D1 is a target gene of miR-195 in cervical cancer cells. DCUN1D1 was predicted as a putative target gene of miR-195 using TargetScan, miRDB, and DIANAmT (Fig. 4A). To confirm this prediction, the WT and MuT DCUN1D1 3'-UTR luciferase reporter plasmids were generated (Fig. 4B). The luciferase reporter gene assay was performed in HeLa and SiHa cells. As presented in Fig. 4C, luciferase activity was significantly reduced in the presence of miR-195 in the HeLa and SiHa cells transfected with WT DCUN1D1 3'-UTR luciferase reporter plasmid, but not those transfected with MuT DCUN1D1 3'-UTR luciferase reporter plasmid.

The effects of miR-195 on the expression levels of DCUN1D1 in cervical cancer cells were evaluated. The data indicated a significant reduction in the mRNA and protein expression levels of DCUN1D1 in miR-195-overexpressing HeLa and SiHa cells (Fig. 4D and E). Thus, DCUN1D1 appears to be the direct target of miR-195 in cervical cancer cells.

Upregulation of DCUN1D1 is associated with the aggressive progression and poor prognosis in cervical cancer. The expression level of DCUN1D1 in cervical cancer and adjacent non-tumor tissue samples was investigated in the current study. The data demonstrate that DCUN1D1 was significantly upregulated in cervical cancer tissue samples when compared with that in adjacent non-tumor tissue samples (Fig. 5A). The correlation between miR-195 and DCUN1D1 expression levels was then evaluated using Pearson's correlation analysis. As presented in Fig. 5B, a significant negative correlation was observed between the expression level of miR-195 and DCUN1D1 in cervical cancer tissue samples. These data indicate that the upregulation of DCUN1D1 may be due to the downregulation of miR-195 in cervical cancer. The clinical significance of DCUN1D1 expression levels was evaluated in cervical cancer. According to the mean value of DCUN1D1 mRNA expression levels (4.14), the cervical cancer patients were divided into a high DCUN1D1 expression level group and a low DCUN1D1 expression level group. As presented in Table II, the upregulation of DCUN1D1 was associated with lymph node metastasis, distant metastasis, and an advanced clinical stage in cervical cancer. Furthermore, the patients with high expression levels of DCUN1D1 exhibited shorter survival times when compared with those with low expression levels of DCUN1D1 (Fig. 5C). Therefore, an increased expression level of DCUN1D1 may predict poor prognosis of patients with cervical cancer.

DCUN1D1 restored miR-195-mediated cell proliferation, migration and invasion in cervical cancer. To confirm miR-195 exerts suppressive effects on cervical cancer cells by targeting DCUN1D1, the miR-195-overexpressing cells were transfected with pcDNA3.1-DCUN1D1 expression plasmid or with blank pcDNA3.1 vector as the control group. Following transfection, the mRNA and protein expression levels of DCUN1D1 were significantly increased in the miR-195 + DCUN1D1 group compared with the miR-195 + blank group (Fig. 6A and B). Furthermore, MTT, wound healing and Transwell assays demonstrated that overexpression of DCUN1D1 restored the suppressive effects of miR-195 on the proliferation, migration and invasion of cervical cancer cells (Figs. 6C and 7). These data confirm that miR-195 exerts inhibitory effects on cervical cancer cells by directly targeting DCUN1D1.

HPV16 E6 regulates the expression levels of miR-195 and DCUNIDI in cervical cancer cells. In the present study, three 

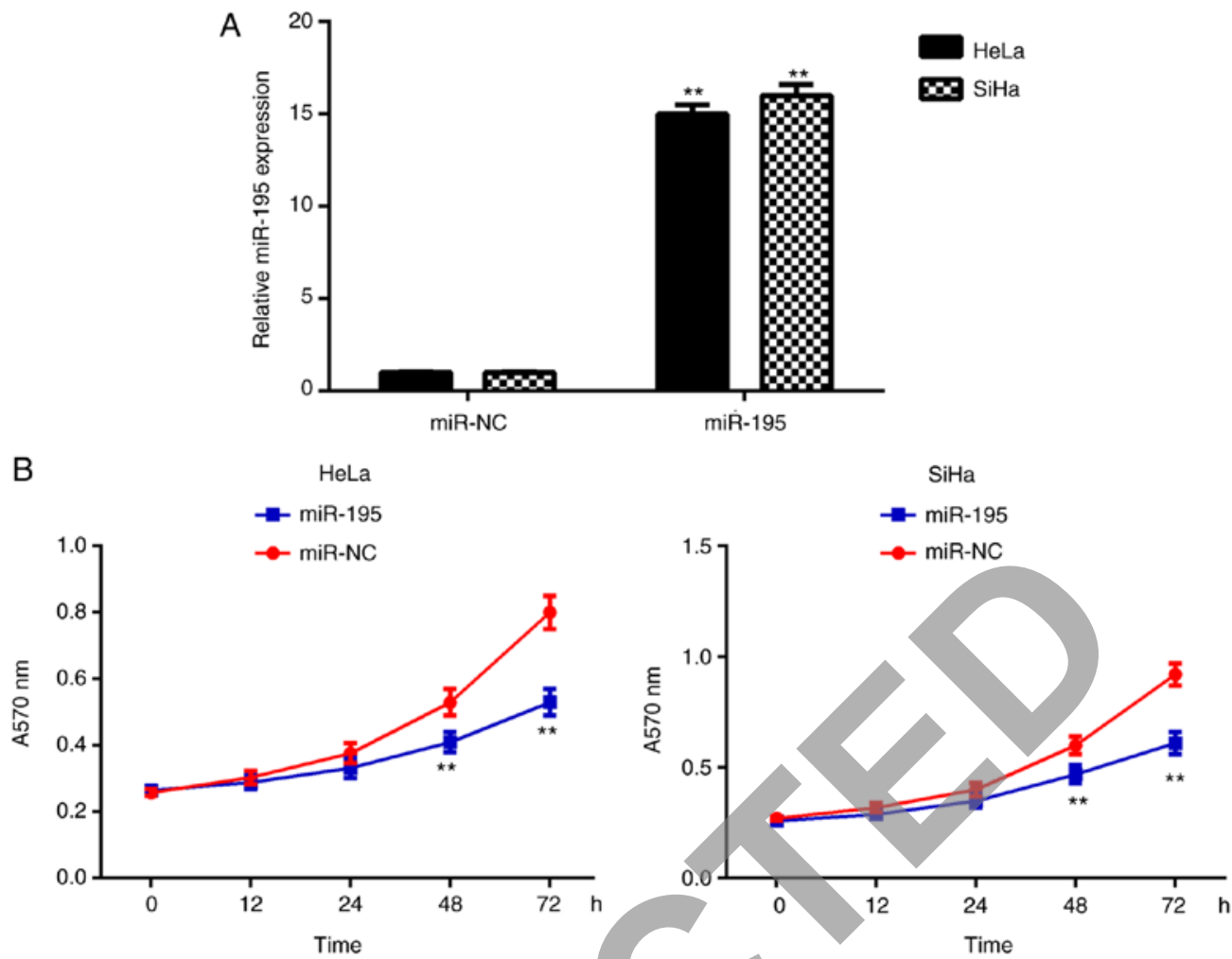

Figure 2. miR-195 exerts suppressive effects on the proliferation of cervical cancer cells. miR-195 mimic or miR-NC was used to transfect HeLa and SiHa cells, respectively. (A) Following transfection, reverse transcription-quantitative polymerase chain reaction was used to evaluate miR-195 expression. (B) MTT assay was performed to examine cell proliferation. ${ }^{* *} \mathrm{P}<0.01$ vs. miR-NC. The experiments were repeated three times. miR, microRNA; miR-NC, negative control miR mimic.

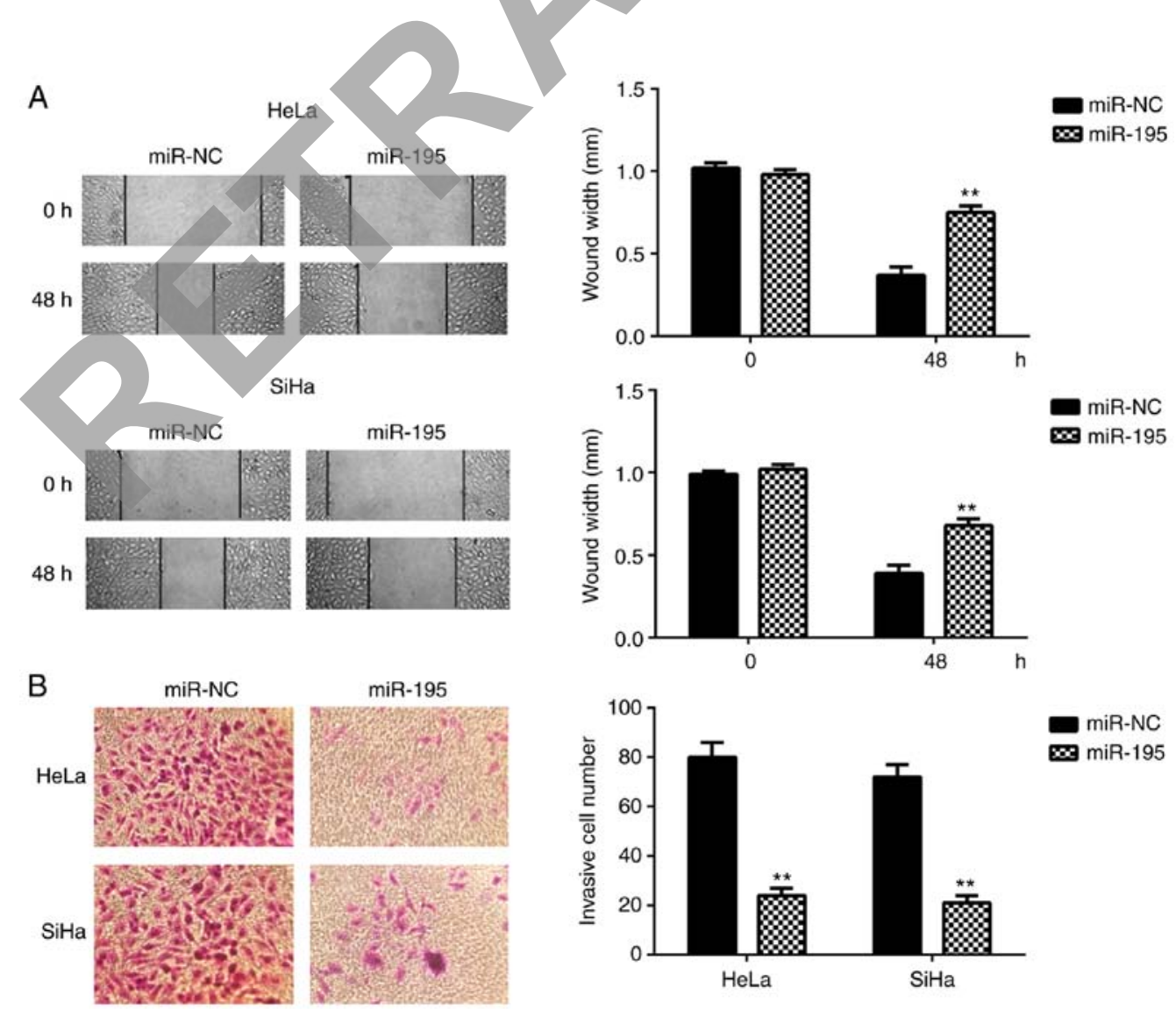

Figure 3. miR-195 has suppressive effects on the migration and invasion of cervical cancer cells. miR-195 mimic or miR-NC was used to transfect HeLa and $\mathrm{SiHa}$ cells, respectively. Following transfection, (A) wound healing (magnification, x40) and (B) Transwell assays (magnification, x400) were performed to

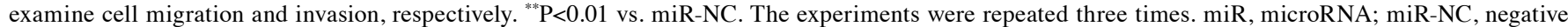
control miR mimic. 


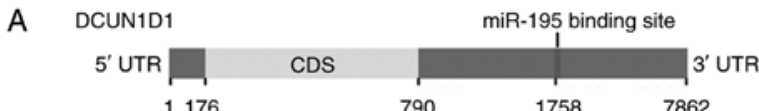

B WT DCUN1D1 $3^{\prime}$ UTR $\quad 5^{\prime}$...AAAAGAAGAAUCUUGCUGCUU.... $3^{\prime}$

miR-195 3 '...CGGUUAUAAAGACACGACGAU...5

Mut DCUN1D1 3' UTR $\quad$ 5'...AAAAGAAGAAUCUACGACGAU...3'
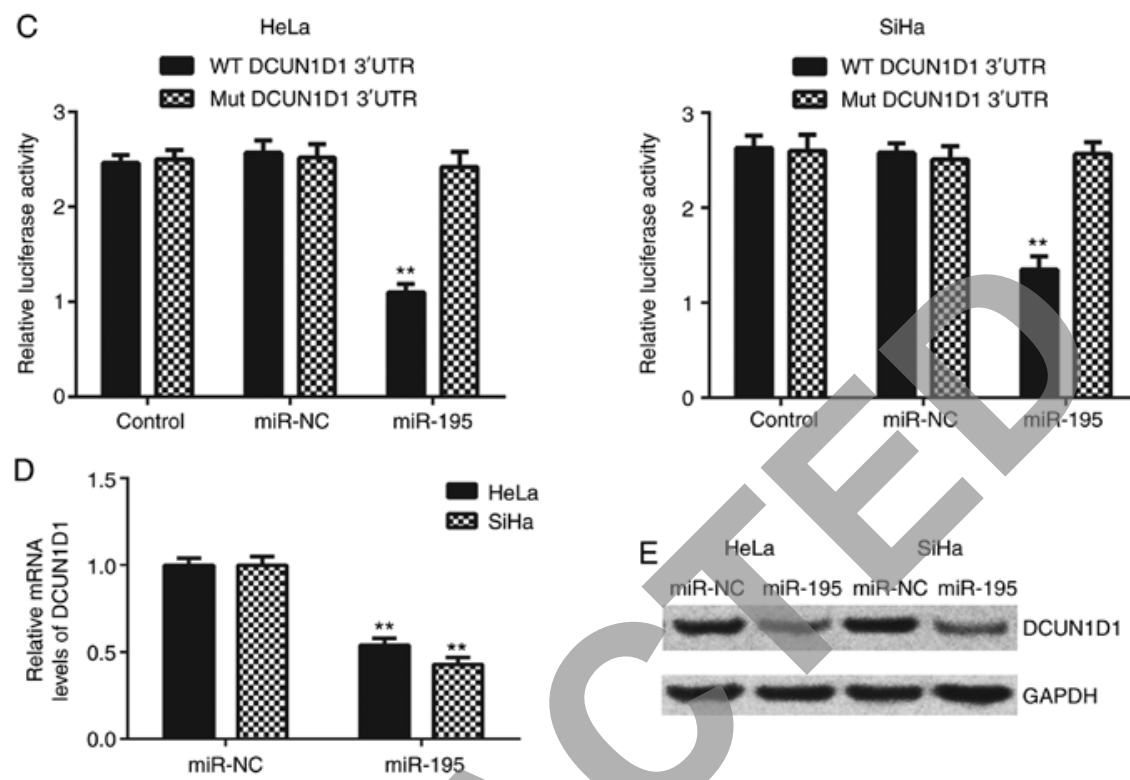

E miR-NC miR-195 miR-NC miR-195

miR-NC miR-195 miR-NC miR-195
DCUN1D1

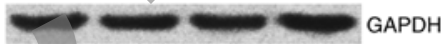

Figure 4. DCUN1D1 is a target gene of miR-195 in cervical cancer cells. (A) DCUN1D1 was predicted to be a putative target gene of miR-195. (B) The WT and MuT DCUN1D1 3'-UTR luciferase reporter plasmids were generated. (C) Luciferase reporter gene assay data indicated that the luciferase activity was significantly reduced in the presence of miR-195 in HeLa and SiHa cells transfected with WT DCUN1D1 3'-UTR luciferase reporter plasmid, but not with MuT DCUN1D1 3'-UTR luciferase reporter plasmid. ${ }^{* *} \mathrm{P}<0.01$ ys. control. (D) Reverse transcription-quantitative polymerase chain reaction and (E) western blotting were used to examine the mRNA and protein expression levels of DCUN1D1 in HeLa and SiHa cells transfected with miR-195 mimic or miR-NC, respectively. ${ }^{* *} \mathrm{P}<0.01$ vs. miR-NC. The experiments were repeated three times. DCUN1D1, defective in cullin neddylation 1 domain containing 1; miR, microRNA; WT, wild-type; MuT, mutant type; miR-NC, negative control miR mimic; CDS, coding sequence; UTR, untranslated region.
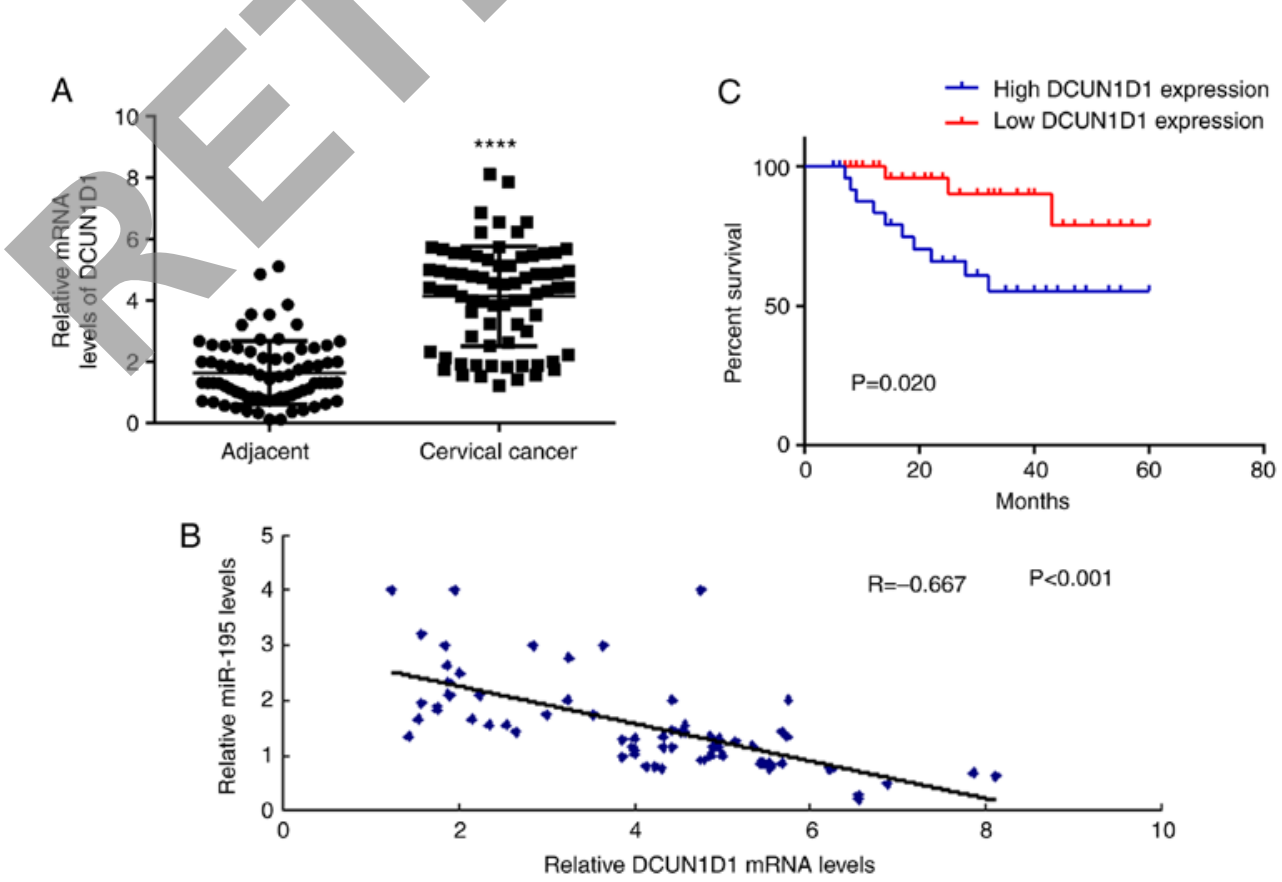

Figure 5. Upregulation of DCUN1D1 is associated with aggressive progression and poor prognosis in cervical cancer. (A) Reverse transcription-quantitative polymerase chain reaction was used to examine the mRNA expression levels of DCUN1D1 in 72 cervical cancer tissue samples compared with their matched adjacent non-tumor tissue samples. ${ }^{* * * * *} \mathrm{P}<0.0001$ vs. adjacent. (B) A significant negative correlation was demonstrated between the expression levels of miR-195 and DCUN1D1 in these 72 cervical cancer tissue samples. (C) The cervical cancer patients with high expression levels of DCUN1D1 exhibited shorter survival times compared with those with low expression levels of DCUN1D1. The experiments were repeated three times. DCUN1D1, defective in cullin neddylation 1 domain containing 1; miR, microRNA; mRNA, messenger RNA. 
Table II. Association between DCUN1D1 expression levels and clinicopathological characteristics of patients with cervical cancer.

\begin{tabular}{|c|c|c|c|c|}
\hline Variable & Patients ( $n=72)$ & Low DCUN1D1 $(n=32)$ & High DCUN1D1 $(n=40)$ & P-value \\
\hline Age (years) & & & & 0.807 \\
\hline$<55$ & 27 & 11 & 16 & \\
\hline$\geq 55$ & 45 & 21 & 24 & \\
\hline Tumor size $(\mathrm{cm})$ & & & & 0.331 \\
\hline$\leq 4$ & 44 & 22 & 22 & \\
\hline$>4$ & 28 & 10 & 18 & \\
\hline Differentiation & & & & 0.105 \\
\hline Well to moderate & 53 & 27 & 26 & \\
\hline Poor & 19 & 5 & 14 & \\
\hline Clinical stage & & & & 0.014 \\
\hline I-II & 47 & 26 & 21 & \\
\hline III-IV & 25 & 6 & 19 & \\
\hline Lymph node metastasis & & & & 0.011 \\
\hline No & 49 & 27 & 22 & \\
\hline Yes & 23 & 5 & 18 & \\
\hline Distant metastasis & & & & 0.035 \\
\hline No & 62 & 31 & 31 & \\
\hline Yes & 10 & 1 & 9 & \\
\hline
\end{tabular}

DCUN1D1, defective in cullin neddylation 1 domain containing 1; miR, microRNA; mRNA, messenger RNA.

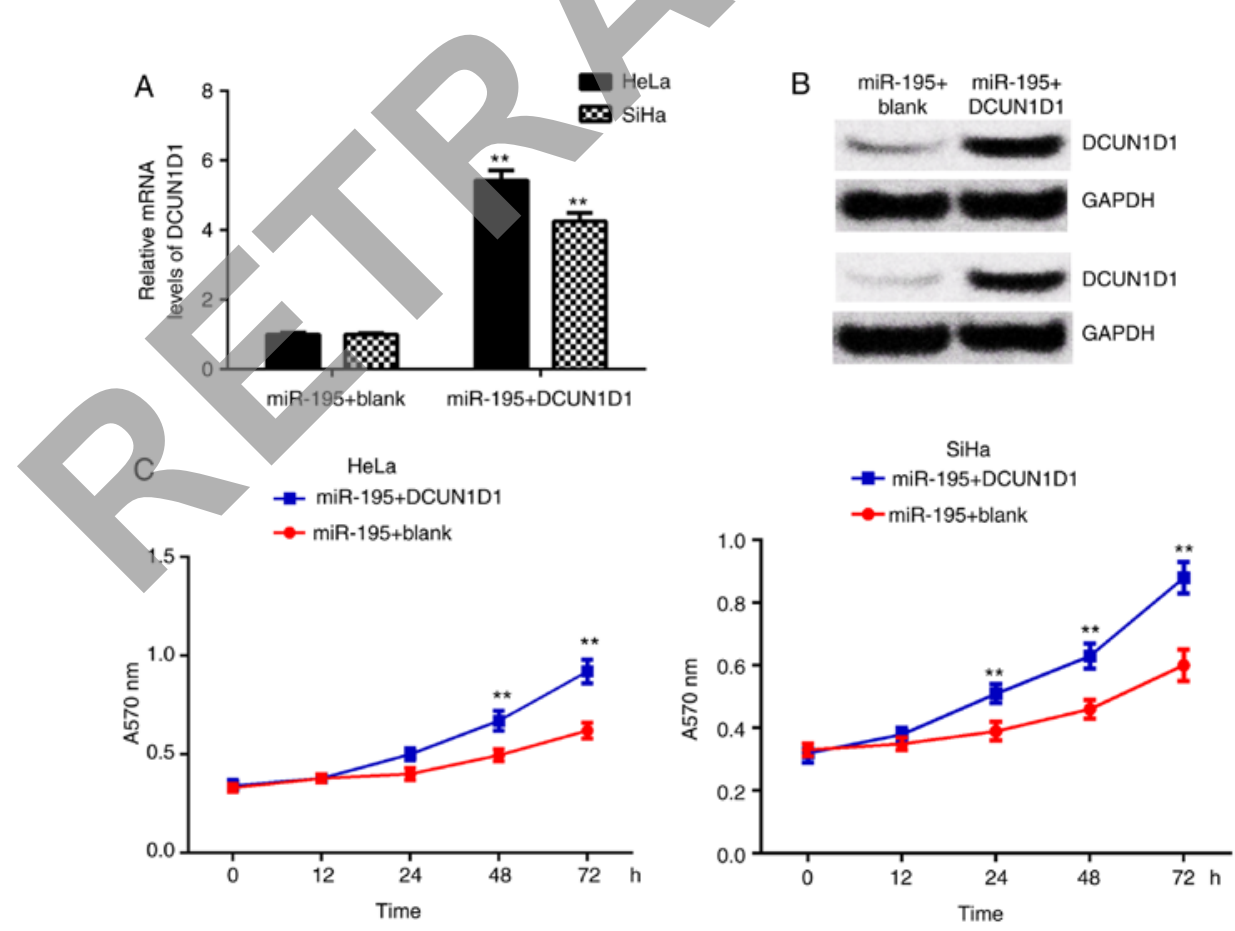

Figure 6. DCUN1D1 restored miR-195-mediated cell proliferation in cervical cancer. The miR-195-overexpressing HeLa and SiHa cells were transfected with pcDNA3.1-DCUN1D1 expression plasmid, or with blank pcDNA3.1 vector as the control group. Following transfection, (A) reverse transcription-quantitative polymerase chain reaction and (B) western blot analysis were performed to examine the mRNA and protein expression levels of DCUN1D1. (C) MTT assay was used to examine cell proliferation. ${ }^{* *} \mathrm{P}<0.01$ vs. miR-195 + blank. The experiments were repeated three times. DCUN1D1, defective in cullin neddylation 1 domain containing 1; miR, microRNA; mRNA, messenger RNA.

common cervical cancer cell lines were used to perform in vitro experiments. C33A cells are $\mathrm{HPV}^{-}$, $\mathrm{SiHa}$ cells are $\mathrm{HPV}^{+} 6^{+}$, and HeLa cells are HPV $18^{+}$. Notably, the qPCR data demonstrated that the expression levels of miR-195 were significantly lower in the HeLa (HPV18 ${ }^{+}$) and $\mathrm{SiHa}\left(\mathrm{HPV}_{16}{ }^{+}\right)$cells, when compared with those in C33A (HPV) cells (Fig. 8A). As HPVs 
A
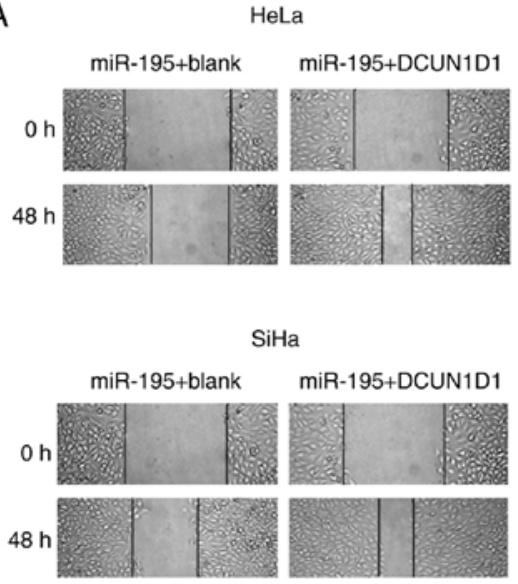

B
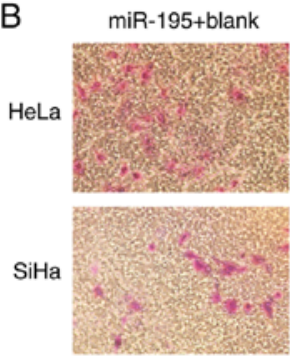

miR-195+DCUN1D1
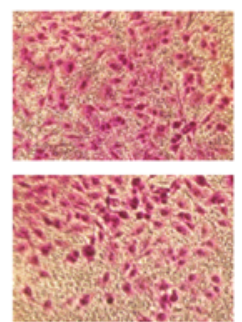
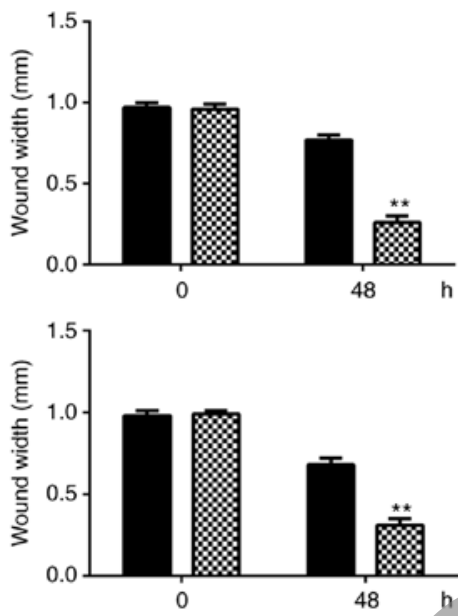

miR-195+blank

$\infty$ miR-195+DCUN1D1

miR-195+blank

$\infty$ miR-195+DCUN1D1
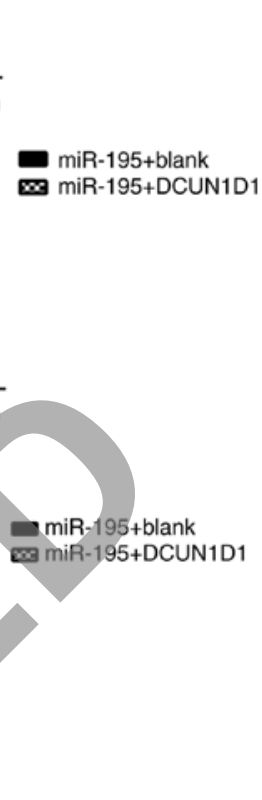

Figure 7. DCUN1D1 restored miR-195-mediated cell migration and invasion in cervical cancer. The miR-195-overexpressing HeLa and SiHa cells were transfected with pcDNA3.1-DCUN1D1 expression plasmid, or with blank pcDNA3.1 vector as the control group. Following transfection, (A) wound healing (magnification, $\mathrm{x} 40$ ) and (B) Transwell (magnification, $\mathrm{x} 400$ ) assays were used to examine cell migration and invasion, respectively. ${ }^{* *} \mathrm{P}<0.01 \mathrm{vs}$. miR-195 + blank. The experiments were repeated three times. DCUN1D1, defective in cullin neddylation 1 domain containing 1; miR, microRNA; mRNA, messenger RNA.

contain two oncogenes (E6 and E7) (18), HPV16 E6, HPV16 E7, HPV18 E6 and HPV18 E7 were knocked down in SiHa or HeLa cells using small interfering (si)RNA. As presented in Fig. 8B, the expression level of miR-195 was significantly increased in SiHa and HeLa cells transfected with E6 siRNA, indicating that E6 is the most important HPV oncoprotein responsible for the inhibition of miR-195 in cervical cancer cells. Consistently, knockdown of E6 significantly reduced the protein expression of DCUN1D1 in SiHa and HeLa cells (Fig. 8C). Therefore, the oncogenic effects of HPV E6 on cervical cancer may be partly via regulating the expression level of miR-195 and, thus, its target gene, DCUN1D1.

\section{Discussion}

The exact regulatory mechanism of miR-195 underlying cervical cancer progression remains largely unclear. In the present study, miR-195 was significantly downregulated in cervical cancer, and a low expression level of miR-195 was associated with malignant progression and poor prognosis in cervical cancer. Ectopic expression of miR-195 inhibited the proliferation, migration and invasion of cervical cancer cells. DCUN1D1, which is significantly upregulated in cervical cancer, was identified as a novel target gene of miR-195, and upregulation of DCUN1D1 was associated with the malignant progression and poor prognosis in cervical cancer. DCUN1D1 demonstrated a negative correlation with miR-195 expression levels in cervical cancer, and overexpression of DCUN1D1 restored the suppressive effects of miR-195 on the malignant phenotypes of cervical cancer cells. Notably, miR-195 was downregulated in HeLa
$\left(\mathrm{HPV} 18^{+}\right)$and $\mathrm{SiHa}\left(\mathrm{HPV} 16^{+}\right)$cells compared with that in C33A (HPV) cells, and knockdown of HPV E6 increased the miR-195 expression level while it reduced the DCUN1D1 expression in $\mathrm{HeLa}$ and SiHa cells.

In recent years, accumulating evidence has demonstrated that miRs are key in tumor initialization, growth, and metastasis by regulating the expression of their specific target genes $(11,20,21)$. Among these cancer-associated miRs, miR-195 typically acts as a tumor suppressor in certain common cancer types, such as gastric (14), colorectal (22) and renal (23) cancer, osteosarcoma (24), and liver cancer (25). Furthermore, recent studies have revealed a role of miR-195 in cervical cancer $(15,17,26)$. Wang et al (26) reported that miR-195 was downregulated in primary cervical cancer tissue samples, and may inhibit the proliferation of cervical cancer cells via targeting cyclin Dla and thus inducing $\mathrm{G}_{1}$ phase arrest. Similarly, Li et al (17) identified that miR-195 suppressed cervical cancer cell proliferation and promoted cell apoptosis by targeting cyclin D1 (17). In the present study, miR-195 was significantly downregulated in cervical cancer tissue samples when compared with adjacent non-tumor tissue samples, and its downregulation was associated with node metastasis and an advanced clinical stage. In addition, Zhou et al (15) observed that the low miR-195 expression level was significantly correlated with a higher clinical stage, node metastasis and deep stromal invasion (15). Furthermore, miR-195 inhibited cervical cancer proliferation and repressed cancer cell migration and invasion in the present study, which was consistent with the findings of previous studies $(15,16)$.

As miRs function by regulating the expression levels of their target genes (7), the target genes of miR-195 were then 

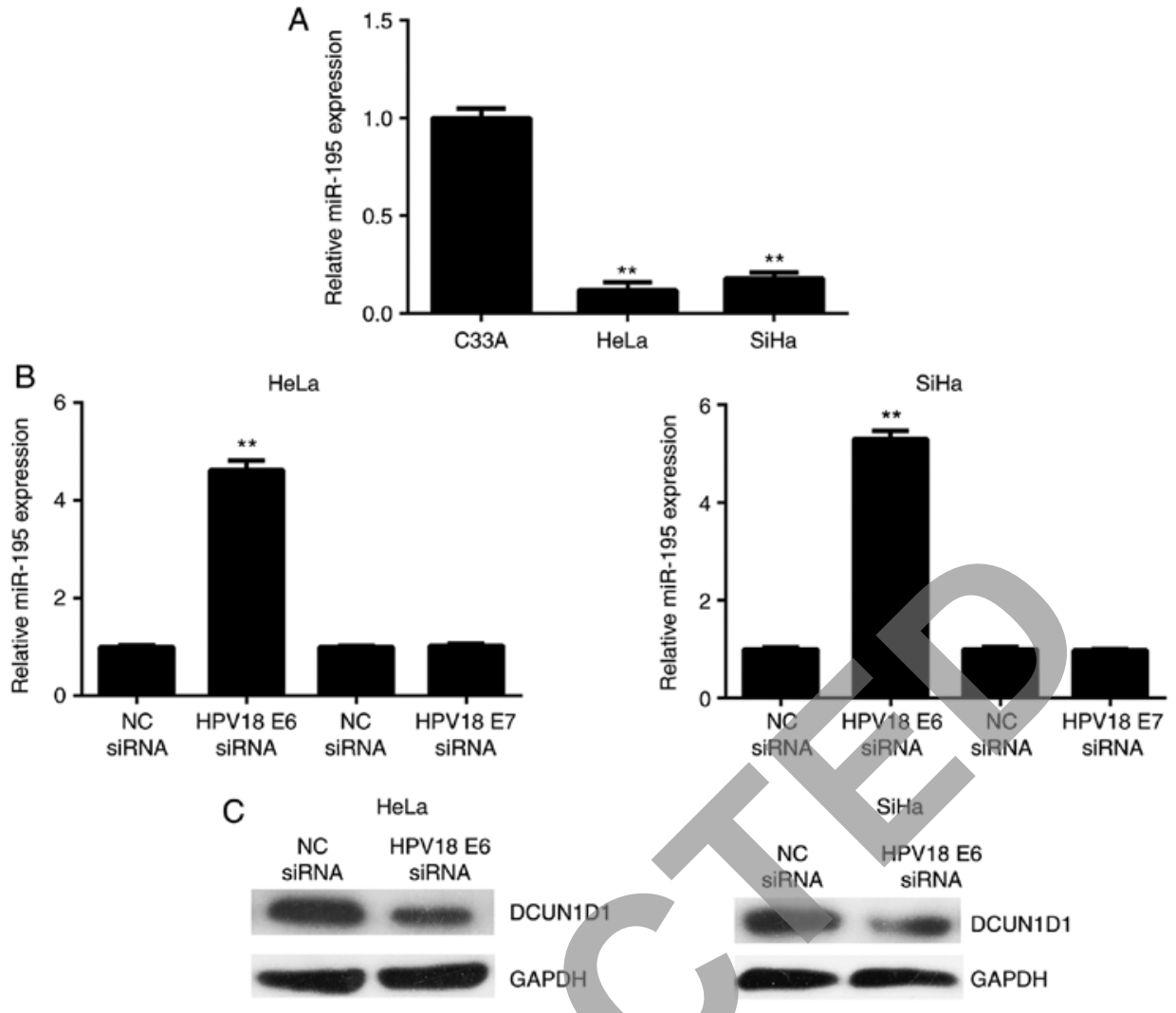

Figure 8. HPV16 E6 regulates the expression levels of miR-195 and DCUN1D1 in cervical cancer cells. (A) Reverse transcription-quantitative polymerase chain reaction was used to examine the miR-195 expression levels in (A) cervical cancer cell lines, including HeLa (HPV18 $)$, SiHa (HPV16 $6^{+}$and C33A (HPV), and (B) HeLa and SiHa cells transfected with HPV16 E6 siRNA, HPV16 E7 siRNA, HPV18 E6 siRNA and HPV18 E7 siRNA, respectively. *P<0.01 vs. C33A. (C) Western blot analysis was performed to examine the protein expression level of DCUN1D1 in SiHa and HeLa cells transfected with HPV16 E6 siRNA or HPV18 E6 siRNA, respectively. The experiments were repeated three times. HPV, human papilloma virus; miR, microRNA; DCUN1D1, defective in cullin neddylation 1 domain containing 1; siRNA, small interfering RNA; NC, negative control.

predicted in cervical cancer cells using bioinformatics analysis. DCUN1D1 was selected as the potential target of miR-195, as a recent study demonstrated that miR-218 inhibits the migration, invasion and epithelial-mesenchymal transition in cervical cancer cells by targeting DCUN1D1 (27). However, whether other miRs may also directly target DCUN1D1 in cervical cancer remains unknown. DCUN1D1, also termed squamous cell carcinoma-related oncogene, may promote nuclear translocation and assembly of the neddylation E3 complex $(28,29)$. Recently, DCUN1D1 was identified to be involved in tumor progression and development of brain metastasis in patients with non-small cell lung cancer (30). Furthermore, DCUN1D1 has been reported to be activated by amplification in squamous cell carcinomas (29). In the current study, DCUN1D1 was significantly upregulated in cervical cancer tissue samples when compared with adjacent normal tissue samples, and its upregulation was significantly associated with node metastasis, distant metastasis, an advanced clinical stage and shorter survival time of patients with cervical cancer. As it was observed that the expression level of DCUN1D1 was negatively correlated to miR-195 expression levels in cervical cancer tissue samples, it was hypothesized that its upregulation may be due to the downregulation of miR-195. Further investigation indicated that DCUN1D1 overexpression restored the suppressive effects of miR-195 on the proliferation, migration and invasion of cervical cancer cells, deonstrating that DCUN1D1 functions as a downstream effecter of miR-195 in cervical cancer cells. In addition to DCUN1D1, various other target genes of miR-195 have been identified in other studies, including Smad3, CCND2, MYB and cyclin D1 (15-17). These target genes have also been proposed to be involved in the miR-195-mediated malignant phenotypes of cervical cancer cells (15-17). For example, Du et al (16) found that miR-195 exerted suppressive effects on cervical cancer cell proliferation, migration and invasion in vitro, and identified CCND2 and MYB as two direct targets of miR-195 in cervical cancer HeLa cells (16).

Notably, the HPV E6 affected the expression levels of miR-195 and DCUN1D1 in cervical cancer cells in the present study. These findings provide a novel potential molecular mechanism in HPV-associated cervical cancer. In addition, the 72 cervical cancer patients that were involved in the present study were high-risk $\mathrm{HPV}^{+}$, and thus no correlation was observed between the miR-195 expression level and HPV infection in these cervical cancer patients.

In conclusion, to the best of our knowledge, this is the first study demonstrating that miR-195 inhibits the proliferation, migration and invasion of cervical cancer cells by directly targeting DCUN1D1. Based on these findings, it is proposed that the miR-195/DCUN1D1 axis may serve as a potential candidate target for the treatment of cervical cancer. A limitation of the present study is that the number of cervical cancer patients is small. Further studies are required to establish the exact role of miR-195 in cervical cancer growth and metastasis in vivo, as well as the downstream signaling pathways. 


\section{Acknowledgements}

Not applicable.

\section{Funding}

No funding was received.

\section{Availability of data and materials}

The datasets generated/analysed during the current study are available.

\section{Authors' contributions}

JZ did most of the experiments and was a major contributor in writing the paper. HY collected clincial tissues and performed the PCR assay. XX performed the statistical analysis.SK designed the study and was also a major contributor in writing the paper. All authors read and approved the final manuscript.

\section{Ethics approval and consent to participate}

The present study was approved by the Ethics Committee of Affiliated Qingdao Hiser Hospital of Qingdao University (Qingdao, China). Written informed consent was obtained from each patient involved in the study.

\section{Consent for publication}

Consent for publication was obtained from the participants.

\section{Competing interests}

The authors declare they have no competing interests.

\section{References}

1. Siegel R, Naishadham D and Jemal A: Cancer statistics, 2012. CA Cancer J Clin 62: 10-29, 2012.

2. Siegel RL, Miller KD and Jemal A: Cancer statistics, 2015. CA Cancer J Clin 65: 5-29, 2015.

3. Li H, Xiang Z, Liu Y, Xu B and Tang J: MicroRNA-133b inhibits proliferation, cellular migration, and invasion via targeting LASP1 in hepatocarcinoma cells. Oncol Res 25: 1269-1282, 2017.

4. Zhu K, He Y, Xia C, Yan J, Hou J, Kong D, Yang Y and Zheng G: MicroRNA-15a inhibits proliferation and induces apoptosis in CNE1 nasopharyngeal carcinoma cells. Oncol Res 24: 145-151, 2016.

5. Jiang Z, Zhang Y, Cao R, Li L, Zhong K, Chen Q and Xiao J: miR-5195-3p inhibits proliferation and invasion of human bladder cancer cells by directly targeting oncogene KLF5. Oncol Res 25: 1081-1087, 2017.

6. Wang G, Fu Y, Liu G, Ye Y and Zhang X: miR-218 inhibits proliferation, migration, and EMT of gastric cancer cells by targeting WASF3. Oncol Res 25: 355-364, 2016.

7. Ambros V: The functions of animal microRNAs. Nature 431: 350-355, 2004

8. Bartel DP: MicroRNAs: Genomics, biogenesis, mechanism, and function. Cell 116: 281-297, 2004.

9. Yao J, Deng B, Zheng L, Dou L, Guo Y and Guo K: miR-27b is upregulated in cervical carcinogenesis and promotes cell growth and invasion by regulating CDH11 and epithelial-mesenchymal transition. Oncol Rep 35: 1645-1651, 2016.

10. Zhang X, Cai D, Meng L and Wang B: MicroRNA-124 inhibits proliferation, invasion, migration and epithelial-mesenchymal transition of cervical carcinoma cells by targeting astrocyte-elevated gene-1. Oncol Rep 36: 2321-2328, 2016.
11. Su Y,Xiong J,Hu J, Wei X,Zhang X and Rao L: MicroRNA-140-5p targets insulin like growth factor 2 mRNA binding protein 1 (IGF2BP1) to suppress cervical cancer growth and metastasis. Oncotarget 7: 68397-68411, 2016.

12. Zeng F, Xue M, Xiao T, Li Y, Xiao S, Jiang B and Ren C: MiR-200b promotes the cell proliferation and metastasis of cervical cancer by inhibiting FOXG1. Biomed Pharmacother 79: 294-301, 2016.

13. Fei X, Qi M, Wu B, Song Y, Wang Y and Li T: MicroRNA-195-5p suppresses glucose uptake and proliferation of human bladder cancer T24 cells by regulating GLUT3 expression. FEBS Lett 586: 392-397, 2012.

14. Wang J, Li L, Jiang M and Li Y: MicroRNA-195 inhibits human gastric cancer by directly targeting basic fibroblast growth factor. Clin Transl Oncol 19: 1320-1328, 2017.

15. Zhou Q, Han LR, Zhou YX and Li Y: MiR-195 suppresses cervical cancer migration and invasion through targeting Smad3. Int J Gynecol Cancer 26: 817-824, 2016.

16. Du X, Lin LI, Zhang L and Jiang J: MicroRNA-195 inhibits the proliferation, migration and invasion of cervical cancer cells via the inhibition of CCND2 and MYB expression. Oncol Lett 10: 2639-2643, 2015

17. Li Z, Wang H, Wang Z and Cai H: MiR-195 inhibits the proliferation of human cervical cancer cells by directly targeting cyclin D1. Tumour Biol 37: 6457-6463, 2016.

18. Livak KJ and Schmittgen TD: Analysis of relative gene expression data using real-time quantitative PCR and the 2(-delta delta C(T)) method. Methods 25: 402-408, 2001.

19. Gómez-Gómez Y, Organista-Nava J and Gariglio P: Deregulation of the miRNAs expression in cervical cancer: Human papillomavirus implications. Biomed Res Int 2013: 407052, 2013.

20. Zhou W, Zou B, Liu L, Cui K, Gao J, Yuan S and Cong N: MicroRNA-98 acts as a tumor suppressor in hepatocellular carcinoma via targeting SALL4. Oncotarget 7: 74059-74073, 2016.

21. Xu X, Zhang Y, Jasper J, Lykken E, Alexander PB, Markowitz GJ, McDonnell DP, Li QJ and Wang XF: MiR-148a functions to suppress metastasis and serves as a prognostic indicator in triple-negative breast cancer. Oncotarget 7: 20381-20394, 2016.

22. Sun M, Song H, Wang S, Zhang C, Zheng L, Chen F, Shi D, Chen Y, Yang C, Xiang Z, et al: Integrated analysis identifies microRNA-195 as a suppressor of Hippo-YAP pathway in colorectal cancer. J Hematol Oncol 10: 79, 2017.

23. Wang K, Sun Y, Tao W, Fei X and Chang C: Androgen receptor (AR) promotes clearcell renal cell carcinoma (ccRCC) migration and invasion via altering the circHIAT1/miR-195-5p/29a-3p/29c-3p/CDC42 signals. Cancer Lett 394: 1-12, 2017.

24. Qu Q, Chu X and Wang P: MicroRNA-195-5p suppresses osteosarcoma cell proliferation and invasion by suppressing naked cuticle homolog 1. Cell Biol Int 41: 287-295, 2017.

25. Zhang H, Zhou D, Ying M, Chen M, Chen P, Chen Z and Zhang F: Expression of long non-coding RNA (lncRNA) small nucleolar RNA host gene 1 (SNHG1) exacerbates hepatocellular carcinoma through suppressing miR-195. Med Sci Monit 22: 4820-4829, 2016

26. Wang N, Wei H, Yin D, Lu Y, Zhang Y, Zhang Q, Ma X and Zhang S: MicroRNA-195 inhibits proliferation of cervical cancer cells by targeting cyclin D1a. Tumour Biol 37: 4711-4720, 2016.

27. Jiang Z, Song Q, Zeng R, Li J, Li J, Lin X, Chen X, Zhang J and Zheng Y: MicroRNA-218 inhibits EMT, migration and invasion by targeting SFMBT1 and DCUN1D1 in cervical cancer. Oncotarget 7: 45622-45636, 2016.

28. Huang G, Kaufman AJ, Ramanathan Y and Singh B: SCCRO (DCUN1D1) promotes nuclear translocation and assembly of the neddylation E3 complex. J Biol Chem 286: 10297-10304, 2011.

29. Sarkaria I, O-charoenrat P, Talbot SG, Reddy PG, Ngai I, Maghami E, Patel KN, Lee B, Yonekawa Y, Dudas M, et al: Squamous cell carcinoma related oncogene/DCUN1D1 is highly conserved and activated by amplification in squamous cell carcinomas. Cancer Res 66: 9437-9444, 2006.

30. Yoo J, Lee SH, Lym KI, Park SY, Yang SH, Yoo CY, Jung JH, Kang SJ and Kang CS: Immunohistochemical expression of DCUN1D1 in non-small cell lung carcinoma: Its relation to brain metastasis. Cancer Res Treat 44: 57-62, 2012.

This work is licensed under a Creative Commons Attribution-NonCommercial-NoDerivatives 4.0 International (CC BY-NC-ND 4.0) License. 